\title{
Electronic transport through single noble gas atoms
}

\author{
L. A. Zotti, ${ }^{1}$ M. Bürkle, ${ }^{2}$ Y. J. Dappe, ${ }^{3}$ F. Pauly, ${ }^{2}$ and J. C. Cuevas ${ }^{1}$ \\ ${ }^{1}$ Departamento de Física Teórica de la Materia Condensada, Universidad Autónoma de Madrid, E-28049 Madrid, Spain \\ ${ }^{2}$ Institut für Theoretische Festkörperphysik and DFG Center for Functional Nanostructures, Karlsruhe Institute of Technology (KIT), \\ D-76131 Karlsruhe, Germany \\ ${ }^{3}$ Institut de Physique et Chimie des Matériaux de Strasbourg (IPCMS), UMR CNRS 7504, 23 rue du Loess, POB 43, \\ F-67034 Strasbourg Cedex, France
}

(Received 28 September 2011; revised manuscript received 27 October 2011; published 10 November 2011)

\begin{abstract}
We present a theoretical study of the conductance of atomic junctions comprising single noble gas atoms (He, $\mathrm{Ne}, \mathrm{Ar}, \mathrm{Kr}$, and $\mathrm{Xe}$ ) coupled to gold electrodes. The aim is to elucidate how the presence of noble gas atoms affects the electronic transport through metallic atomic-size contacts. Our analysis, based on density functional theory and including van der Waals interactions, shows that for the lightest elements ( $\mathrm{He}$ and $\mathrm{Ne}$ ) no significant current flows through the noble gas atoms and their effect is to reduce the conductance of the junctions by screening the interaction between the gold electrodes. This explains the observations reported in metallic atomic-size contacts with adsorbed $\mathrm{He}$ atoms. Conversely, the heaviest atoms ( $\mathrm{Kr}$ and $\mathrm{Xe}$ ) increase the conductance because of the additional current path provided by their valence $p$ states.
\end{abstract}

DOI: 10.1103/PhysRevB.84.193404

PACS number(s): 73.63.Rt, 73.40.Gk, 73.40.Jn

Noble gases are commonly employed in scanning probe experiments as exchange gases since they are expected to interact weakly with the studied systems. Furthermore, it is often assumed that the adsorption of noble gas (NG) atoms does not affect the electron tunneling between metallic electrodes. However, it has been shown that this is not entirely true. For instance, two decades ago Eigler and coworkers presented scanning tunneling microscope (STM) images of Xe atoms on a $\mathrm{Ni}(110)$ surface $^{1}$ and they nicely demonstrated that these atoms can be moved to chosen positions on the surface. It has also been shown that it is possible to manipulate individual $\mathrm{Xe}$ atoms to construct atomic wires and to measure their electrical resistance ${ }^{2}$ or to functionalize molecules. ${ }^{3}$

From the theoretical side, while there are numerous works analyzing the interaction between $\mathrm{NG}$ atoms and metal surfaces, studies exploring the transport through metal-NGmetal junctions are rather scarce, and most of them have focused either on understanding atomic manipulation or on STM imaging. ${ }^{2,4-8}$ There are still important open problems concerning how adsorbed NG atoms modify the transport through metallic atomic-size junctions. A striking example is the observation made in several break-junction and STM experiments that adsorbed $\mathrm{He}$ atoms can strongly modify the current through metallic junctions, lowering in particular the low-bias conductance. ${ }^{9-12}$ This conductance suppression is surprising since the height of the tunneling barrier in the presence of NG atoms has been predicted to decrease, $;$ indeed, Kelvin probe experiments have shown that the work function of noble metal surfaces decreases upon adsorption of $\mathrm{Ar}, \mathrm{Kr}$, and $\mathrm{Xe} .{ }^{13}$ A possible explanation, based on predictions by Lang, ${ }^{4}$ suggests that adsorbed $\mathrm{He}$ atoms can polarize metal states away from the Fermi energy, leading to a decrease in the metal local density of states. This explanation was based on calculations in which the metal electrodes were described by a jellium model (with no atomistic details) and without taking into account van der Waals interactions. Thus, it is highly desirable to revisit this problem with ab initio transport methods.
To shed new light on the influence of adsorbed NG atoms in the transport through metallic atomic contacts, we present in this Brief Report a systematic ab initio study of the conductance of gold atomic junctions containing single atoms of $\mathrm{He}, \mathrm{Ne}, \mathrm{Ar}, \mathrm{Kr}$, and Xe. Our calculations, based on density functional theory (DFT), show that, while for $\mathrm{He}$ and $\mathrm{Ne}$ the current flows directly from one metallic electrode to the other, for $\mathrm{Ar}, \mathrm{Kr}$, and $\mathrm{Xe}$ the transport occurs mainly through the valence $p$ states of the NG atom. In all cases, the presence of NG atoms induces a dipole moment which screens the interaction between the leads. In the case of $\mathrm{He}, \mathrm{Ne}$, and Ar the weakening of the metal-metal coupling (rather than a suppression of the metal density of states, as proposed by $\mathrm{Lang}^{4}$ ) leads to a reduction of the tunneling current. On the contrary, for $\mathrm{Kr}$ and $\mathrm{Xe}$ the additional tunneling path provided by the valence $p$ states overcomes the screening, leading to an enhancement of the current.

Our main goal is to analyze the electronic transport through metallic atomic-size contacts containing single atoms of noble gases. In particular, we have chosen gold for the electrode material and studied the elements $\mathrm{He}, \mathrm{Ne}, \mathrm{Ar}, \mathrm{Kr}$, and $\mathrm{Xe}$. For this purpose, we have carried out conductance calculations within the framework of DFT following the method described in Ref. 14, which is built upon the TURBOMOLE 6.1 code. ${ }^{15}$ In all our calculations we have used the BP86 functional. ${ }^{16}$ The first step in our analysis is the construction of the atomic junctions. This is done by optimizing geometries where the gold electrodes are formed by two finite clusters of 20 atoms and a single $\mathrm{NG}$ atom is placed in the middle. In the optimization, the $\mathrm{NG}$ atom and the four innermost gold atoms on each side were relaxed, while the other gold atoms were kept frozen. For the optimized atoms, a def2-TZVP basis set ${ }^{17}$ was chosen, while a def-SVP basis set ${ }^{18}$ was used for the frozen gold atoms. The binding energies calculated in this way were found to differ by only around $5 \times 10^{-4} \mathrm{eV}$ from those calculated with a def2-TZVP basis set for all the atoms. Subsequently, the gold cluster size was extended to 116 atoms on each side in order to correctly describe the metal-NG atom charge transfer 


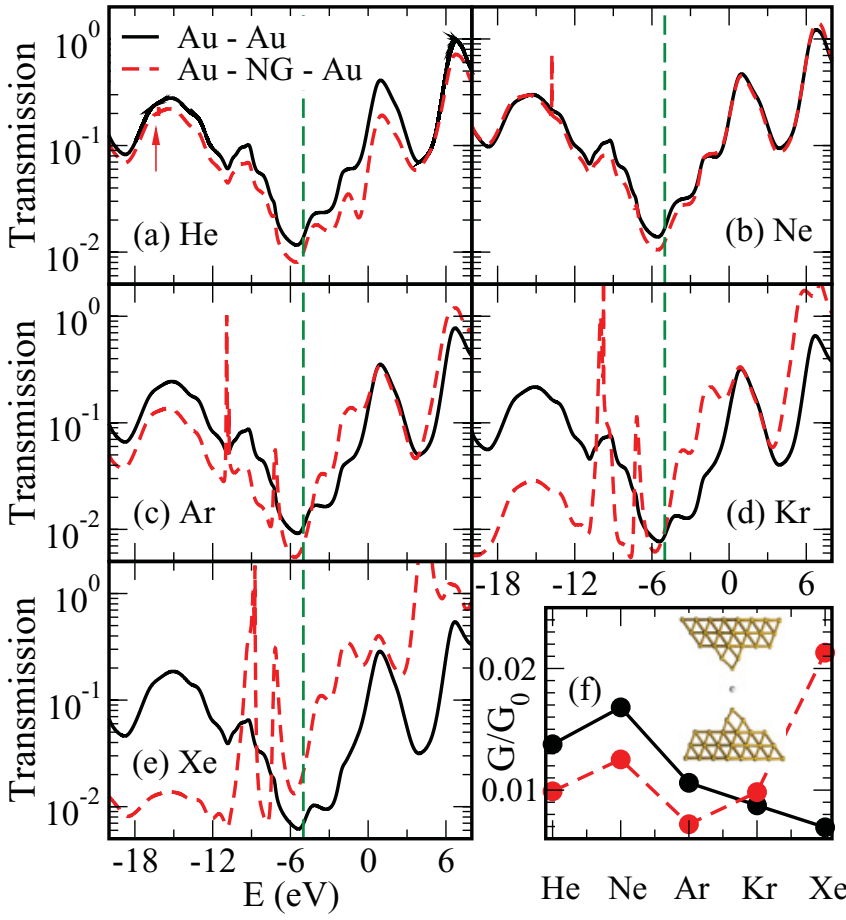

FIG. 1. (Color online) (a)-(e) Zero-bias total transmission as a function of energy for Au-Au (solid black) and Au-NG-Au (dashed red) junctions in the top binding geometry, as shown in the inset of panel (f). The vertical dashed lines indicate the gold Fermi level $(-5 \mathrm{eV})$. (f) Comparison of conductances for $\mathrm{Au}-\mathrm{Au}$ and $\mathrm{Au}-\mathrm{NG}-\mathrm{Au}$ junctions for all studied NG atoms.

and the energy level alignment [see inset in Fig. 1(f)]. It is important to emphasize that we have used the semiempirical DFT-D2 correction ${ }^{19}$ in order to take the dispersive forces into account, since the binding distance between NGs and noble metals is known to be determined by the interplay between the Pauli repulsion and the van der Waals interaction..$^{20,21}$ Finally, the information about the electronic structure of the junctions obtained with DFT is transformed into linear conductance using Green's function techniques as described in detail in Ref. 14. This is done in the spirit of the Landauer approach, where the low-temperature linear conductance is given by $G=G_{0} T\left(E_{\mathrm{F}}\right)=G_{0} \sum_{i} \tau_{i}\left(E_{\mathrm{F}}\right)$, where $G_{0}=2 e^{2} / h$ is the quantum of conductance, $T\left(E_{\mathrm{F}}\right)$ is the total transmission of the junction at the Fermi energy $E_{\mathrm{F}}$, and $\left\{\tau_{i}\right\}$ are the transmission coefficients, i.e., the eigenvalues of the transmission matrix.

We now start our analysis of the results by comparing the linear conductance of gold junctions containing the five NG atoms considered in this work. We consider first junctions with a top binding position [see inset in Fig. 1(f)], since such a geometry has been suggested as the most favorable for most of the NG atoms on metal surfaces. ${ }^{20,22-25}$ The Au-NG-atom distances and the corresponding binding energies are listed in Table I. In particular, the binding energies increase as we move to heavier elements, in agreement with the calculations of Pershina et al. ${ }^{20}$ In Figs. 1(a)-1(e) we show the zero-bias transmission as a function of energy for the five Au-NG-Au junctions with top binding geometries (dashed lines). Notice that below $E_{\mathrm{F}}$ (marked by a vertical dashed line), pronounced peaks appear, which move toward the Fermi energy as the
TABLE I. Energies of the highest occupied $\left(\epsilon_{h}\right)$ and lowest unoccupied $\left(\epsilon_{l}\right)$ states for the NG atoms in the gas phase, plus the calculated quantities for the top-binding geometries with a single gold cluster of 116 atoms: binding distance, dipole moment, charge on the NG atom, and binding energy. The value of the dipole in the bare Au cluster is 1.10 debye. Concerning the corresponding quantities in the junction, the binding distances do not change, while the charges on the NG atom and the binding energies are approximately doubled.

\begin{tabular}{ccccccc}
\hline \hline & & & $\begin{array}{c}\text { Au-NG } \\
\text { distance } \\
(\AA)\end{array}$ & $\begin{array}{c}\text { Dipole } \\
\text { moment } \\
(\text { debye })\end{array}$ & $\begin{array}{c}\text { Charges on } \\
\text { NG atom } \\
(|e|)\end{array}$ & $\begin{array}{c}\text { Binding } \\
\text { energy } \\
(\mathrm{eV})\end{array}$ \\
\hline $\mathrm{He}$ & -15.79 & 16.69 & 3.41 & 1.26 & 0.019 & -0.01 \\
$\mathrm{Ne}$ & -13.29 & 14.99 & 3.28 & 1.38 & 0.018 & -0.05 \\
$\mathrm{Ar}$ & -10.30 & 10.20 & 3.55 & 1.66 & 0.033 & -0.09 \\
$\mathrm{Kr}$ & -9.36 & 6.81 & 3.63 & 1.76 & 0.028 & -0.13 \\
$\mathrm{Xe}$ & -8.32 & 4.69 & 3.73 & 2.11 & 0.028 & -0.18 \\
\hline
\end{tabular}

atomic number of the NG atom increases. These peaks appear approximately at the energies of the highest occupied states of the NG atoms in the gas phase (see Table I), which suggests that they originate from the valence $p$ states for $\mathrm{Ne}, \mathrm{Ar}, \mathrm{Kr}$, and $\mathrm{Xe}$, and from the $1 s$ state for He. A closer look at the peaks for $\mathrm{Ne}, \mathrm{Ar}, \mathrm{Kr}$, and Xe shows that they are split into two. One corresponds to the $p_{z}$ orbital ( $z$ being the direction of the junction axis), which is shifted to lower energies, and the second is due to the $p_{x}$ and $p_{y}$ states, which remain degenerate in the junction. ${ }^{26}$ The peak or resonance due to the $p_{z}$ orbital is clearly broader due to its stronger hybridization with the gold states. Moreover, the width of this resonance increases from $\mathrm{Ne}$ to Xe simply because it is determined by the local density of states (LDOS) of the gold tip atoms at the energy of the valence states of the NG atoms. For $\mathrm{Ne}$ and $\mathrm{Ar}$, that energy lies outside the $5 d$ band of gold, while for $\mathrm{Kr}$ and $\mathrm{Xe}$, it is well inside this band. Regarding the conductance, displayed in Fig. 1(f), it varies in a nonmonotonic manner from $10^{-2} G_{0}$ for He to approximately $2.1 \times 10^{-2} G_{0}$ for Xe. Notice that the computed conductance for $\mathrm{Xe}$ is lower by one order of magnitude than that measured in Ref. 2. We attribute this to the different electrode material (Au rather than $\mathrm{Ni}$ ).

To understand the mechanism governing the conduction through the NG atoms and how they modify the transport through the gold junctions, we also present in Figs. 1(a)-1(e) the transmission curves for Au-Au junctions (with no NG atoms) and in Fig. 1(f) the corresponding conductance. In these calculations we have kept the gold electrodes at the same distance as in the corresponding Au-NG-Au junctions. The first thing to notice is that for the lightest elements the conductance is lowered when the NG atoms are in the junctions. As explained in the Introduction, such a reduction of the conductance caused by the adsorption of He has been observed in several low-temperature break-junction and STM experiments. ${ }^{9} 10,12$ A similar behavior has been observed for closed-shell molecules such as $\mathrm{H}_{2}{ }^{32}$ For $\mathrm{He}$ and $\mathrm{Ne}$, we find that the conductance is decreased by about $30 \%$ when these atoms are present. On the contrary, the presence of $\mathrm{Kr}$ and $\mathrm{Xe}$ in the junctions gives rise to an increase of the conductance, which is larger than a factor of 2 for Xe. What is the reason for 
this different behavior? A first hint is obtained by comparing the transmission curves of the junctions with and without NG atoms. As one can see in Figs. 1(a) and 1(b), for $\mathrm{He}$ and $\mathrm{Ne}$ the transmission curves for the Au-NG-Au junctions follow very closely the energy dependence of the $\mathrm{Au}-\mathrm{Au}$ junctions. This fact suggests that for $\mathrm{He}$ and $\mathrm{Ne}$, the main contribution to the current in the $\mathrm{Au}-\mathrm{NG}-\mathrm{Au}$ junctions comes from direct tunneling from gold to gold, and the only effect of these NG atoms is to reduce the transmission of the gold-to-gold current path. On the other hand, for $\mathrm{Ar}, \mathrm{Kr}$, and $\mathrm{Xe}$, the transmission curves differ markedly from those of the Au-Au junctions, especially close to the Fermi energy. This suggests that the valence $p$ states of these atoms are contributing significantly to the transport through these junctions.

The fact that $\mathrm{He}$ and $\mathrm{Ne}$ do not contribute to the electronic transport is reasonable, as their highest occupied states lie very far away from the Fermi level and they are weakly coupled to the electrodes. The conductance suppression is, however, surprising, as it is, a priori, not obvious how a closed-shell atom can conduct less than the vacuum. We explain this suppression as follows. In our analysis of the interaction between a NG atom and a single gold cluster we have found that there is a tiny charge transfer (a fraction of an electron leaves the NG atoms), which induces a dipole moment pointing away from the metal. ${ }^{27}$ The values of the charge transfers and the dipole moments are reported in Table I. The existence of this dipole moment has been predicted by numerous authors in the

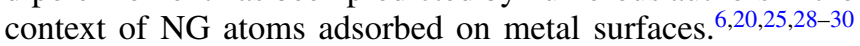
Also in that context, it has been argued that as a consequence of the induced dipole the charge is pushed from the interstitial vacuum region toward the $\mathrm{Au}$ bulk (the so-called pillow or cushion effect). In our case, we did not find a notable charge depletion on the gold tips. We also did not find any significant change in the LDOS of the gold tips at the Fermi level, as was proposed by $\mathrm{Lang}^{4}$ (see Ref. 26) or more recently by Weiss et al. ${ }^{32}$ for the $\mathrm{H}_{2}$ case. Therefore, we suggest that, at least for $\mathrm{He}$ and $\mathrm{Ne}$, what causes the current suppression is the induced polarization which decreases the coupling between the two leads, reducing the transmission of the direct gold-to-gold path. The discrepancy between our results and those of Ref. 4 could be due to the limitations of the jellium model used in that work.

Convincing evidence of the validity of our arguments above is provided by the analysis of the conduction channels. In the left panels of Fig. 2 we show both the total transmission and the individual transmission coefficients $\left\{\tau_{i}\right\}$ as a function of energy for the $\mathrm{Au}-\mathrm{Au}, \mathrm{Au}-\mathrm{He}-\mathrm{Au}$, and $\mathrm{Au}-\mathrm{Xe}-\mathrm{Au}$ junctions. In the $\mathrm{Au}-\mathrm{Au}$ junction the interelectrode distance is the same as in the $\mathrm{Au}-\mathrm{He}-\mathrm{Au}$ junction. In all cases, the transmission at the Fermi energy is dominated by a single channel. More importantly, the transmission coefficients are very similar for the $\mathrm{Au}-\mathrm{Au}$ and the $\mathrm{Au}-\mathrm{He}-\mathrm{Au}$ junctions, suggesting a common transport mechanism, whereas for the $\mathrm{Au}-\mathrm{Xe}-\mathrm{Au}$ junction new features are visible around $E_{\mathrm{F}}$, originating from valence $p$ states of the $\mathrm{Xe}$ atom. The nature of the dominant channel at $E_{\mathrm{F}}$ can be established by looking at the corresponding wave functions of this eigenchannel. Such (right-moving) wave functions for these three junctions are shown in the right panels of Fig. 2. They have been calculated using the method described in Ref. 31. Notice that there is no weight present in the gap

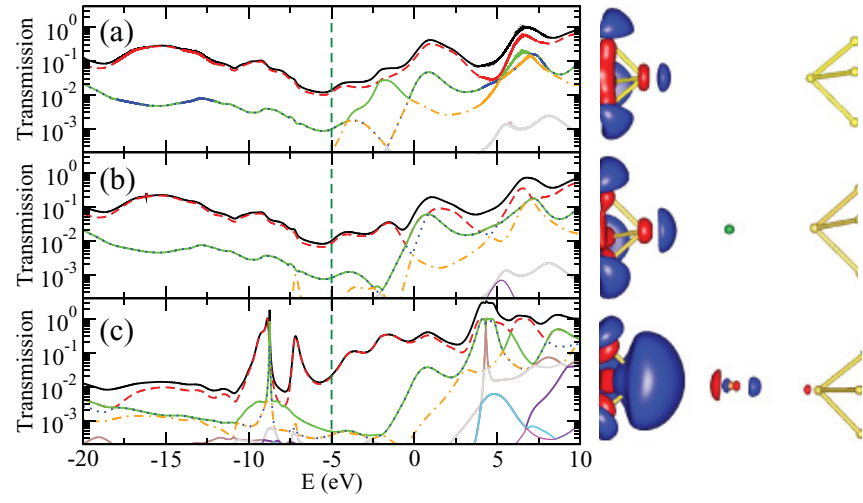

FIG. 2. (Color online) The left panels show the total transmission (solid lines) and the largest transmission coefficients (dashed lines) as a function of energy for (a) $\mathrm{Au}-\mathrm{Au}$, (b) $\mathrm{Au}-\mathrm{He}-\mathrm{Au}$, and (c) $\mathrm{Au}-$ $\mathrm{Xe}-\mathrm{Au}$ junctions. The transmission coefficients have been classified according to the magnitude of the eigenvalue. The right panels show the corresponding right-moving wave functions of the dominant eigenchannel at the Fermi energy for the same isosurface values.

region either in the $\mathrm{Au}-\mathrm{Au}$ junctions, as expected, or in the $\mathrm{Au}-\mathrm{He}-\mathrm{Au}$ one. On the other hand, in the $\mathrm{Au}-\mathrm{Xe}-\mathrm{Au}$ junction the channel in the central region has the symmetry of the $\mathrm{Xe}$ $p_{z}$ orbital, demonstrating that this orbital plays the main role in the conduction through this junction, as hinted in Ref. 33. This is at variance with the calculations of Ref. 7, where the transport through Xe was found to occur through the tail of the $6 s$ orbital.

We have checked that the qualitative behavior of the different NG atoms is also exhibited in other binding geometries. An example of $\mathrm{Au}-\mathrm{He}-\mathrm{Au}$ and $\mathrm{Au}-\mathrm{Xe}-\mathrm{Au}$ junctions with hollow binding geometries is shown in Ref. 26. On the other hand, one may wonder whether the special behavior of the $\mathrm{He}$ is sensitive to the interelectrode distance. This is an important question since experimentally it is not easy to determine absolute distances, and the influence of the $\mathrm{He}$ atoms has been deduced from the conductance decay when the junctions are stretched toward the tunnel regime. To answer this question, we have simulated the stretching of a $\mathrm{Au}-\mathrm{He}-\mathrm{Au}$ junction starting from the equilibrium geometry of Fig. 1. For these calculations we have modeled the narrowest part

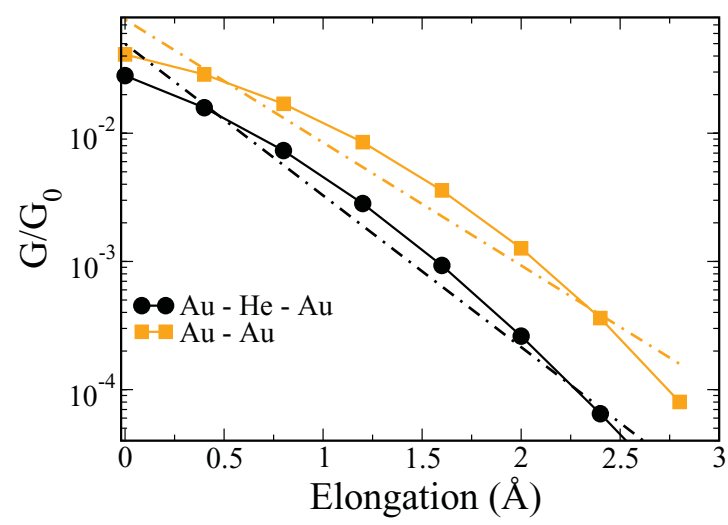

FIG. 3. (Color online) Conductance vs elongation for $\mathrm{Au}-\mathrm{Au}$ and $\mathrm{Au}-\mathrm{He}-\mathrm{Au}$ junctions upon stretching (solid lines) and fitting curves to an exponential function (dashed-dotted lines), see text. 
of the electrodes with clusters of 20 gold atoms, and have checked that the results are consistent with those obtained with the larger clusters. In Fig. 3 we show the evolution of the conductance of the Au-He-Au junction upon separating the gold electrodes symmetrically from the He atom in the equilibrium position. For comparison we also include the results for the corresponding $\mathrm{Au}-\mathrm{Au}$ junction. Figure 3 shows that the presence of the He atom suppresses the conductance also at larger distances. The conductance decay for both junctions can be roughly fitted with an exponential function $G=A e^{-\beta d}$, where $\beta$ is the attenuation factor and $d$ corresponds to the elongation, as measured with respect to the equilibrium position. The attenuation factor $\beta$ is equal to $2.72 \AA^{-1}$ for the junction with the $\mathrm{He}$ atom and $2.21 \AA^{-1}$ for the pure gold junction. The faster decay with the He atom is consistent with the experiments, $, 10,12$ and it shows that the conductance suppression can be more dramatic at large distances (up to $82 \%$ ).

In summary, we have presented a theoretical analysis of the influence of NG atoms on the conductance of gold atomic contacts. We have shown that $\mathrm{He}$ and $\mathrm{Ne}$ atoms do not conduct and their effect is to reduce the conductance of the metallic junctions due to polarization effects. As we consider the tunneling current through heavier noble gas atoms, the polarization effects increase but they are compensated for by an increasing contribution of the valence $p$ states of the NG atoms, leading to an enhancement of the conductance. Our study shows that the presence of absorbed NG atoms can modify significantly the conduction through atomic-scale junctions, which has important implications for a great variety of experiments in the field of molecular electronics.

We thank E. Leary, N. Agraï, and T. Frederiksen for useful discussions. L.A.Z. and J.C.C. were funded by the EU through BIMORE (MRTN-CT-2006-035859) and by the Comunidad de Madrid through the program NANOBIOMAGNET S2009/MAT1726. M.B acknowledges funding through the Center for Functional Nanostructures and the DFG priority program 1243, and F.P. through the Young Investigator Group.
${ }^{1}$ D. M. Eigler and E. K. Schweizer, Nature (London) 344, 524 (1990); D. M. Eigler, P. S. Weiss, E. K. Schweizer, and N. D. Lang, Phys. Rev. Lett. 66, 1189 (1991).

${ }^{2}$ A. Yazdani, D. M. Eigler, and N. D. Lang, Science 272, 1921 (1996).

${ }^{3}$ X. Qiu et al., J. Am. Chem. Soc. 124, 14804 (2002).

${ }^{4}$ N. D. Lang, Phys. Rev. Lett. 56, 1164 (1986).

${ }^{5}$ J. Mahanty and M. Tsukada, Surf. Sci. Lett. 258, L679 (1991).

${ }^{6}$ J. J. Saenz and N. García, Phys. Rev. B 47, 7537 (1993).

${ }^{7}$ N. D. Lang, Surf. Sci. 299, 284 (1994).

${ }^{8}$ F. Flores et al., Prog. Surf. Sci. 48, 27 (1995); N. Mingo et al., Phys. Rev. B 54, 2225 (1996); S. Gao, M. Persson, and B. I. Lundqvist, ibid. 55, 4825 (1997); X. Bouju et al., ibid. 55, 16498 (1997); L. Pizzagalli et al., Appl. Phys. A 66, S875 (1998); M. Hliwa and C. Joachim, Phys. Rev. B 65, 085406 (2002).

${ }^{9}$ R. J. P. Keijsers et al., Phys. Rev. Lett. 76, 1138 (1996).

${ }^{10}$ R. J. P. Keijsers, O. I. Shklyarevskii, and H. van Kempen, Phys. Rev. B 61, 7328 (2000).

${ }^{11}$ O. Yu. Kolesnychenko, O. I. Shklyarevskii, and H. van Kempen, Phys. Rev. Lett. 83, 2242 (1999).

${ }^{12}$ C. Untiedt et al., Phys. Rev. B 66, 085418 (2002).

${ }^{13}$ C. Hückstädt et al., Phys. Rev. B 73, 075409 (2006).

${ }^{14}$ F. Pauly et al., New J. Phys. 10, 125019 (2008).

${ }^{15}$ R. Ahlrichs et al., Chem. Phys. Lett. 162, 165 (1989).
${ }^{16}$ J. P. Perdew, Phys. Rev. B 33, 8822 (1986).

${ }^{17}$ F. Weigend et al., Phys. Chem. Chem. Phys. 7, 3297 (2005).

${ }^{18}$ A. Schäfer et al., J. Chem. Phys. 97, 2571 (1992).

${ }^{19}$ S. Grimme, J. Comput. Chem. 27, 1787 (2006).

${ }^{20}$ V. Pershina et al., J. Chem. Phys. 129, 144106 (2008).

${ }^{21}$ The actual bonding nature is still under debate, see for instance, J. E. Müller, Appl. Phys. A 87, 433 (2007).

${ }^{22}$ M. Petersen et al., Phys. Rev. Lett. 76, 995 (1996).

${ }^{23}$ R. D. Diehl et al., J. Phys. Condens. Matter 16, S2839 (2004).

${ }^{24}$ J. L. F. Da Silva et al., Phys. Rev. B 72, 075424 (2005).

${ }^{25}$ J. L. F. Da Silva and C. Stampfl, Phys. Rev. B 77, 045401 (2008).

${ }^{26}$ See Supplemental Material at http://link.aps.org/supplemental/ 10.1103/PhysRevB.84.193404 for further details.

${ }^{27}$ The total dipole moment vanishes in the Au-NG-Au junctions because of the symmetry of the contacts.

${ }^{28}$ J. E. Müller, Phys. Rev. Lett. 65, 3021 (1990).

${ }^{29}$ A. Widom et al., Phys. Rev. B 62, 16085 (2000).

${ }^{30}$ M. I. Trioni et al., J. Phys. Condens. Matter 21, 264003 (2009).

${ }^{31}$ M. Paulsson and M. Brandbyge, Phys. Rev. B 76, 115117 (2007); M. Bürkle et al., e-print arXiv:1109.0273v1 [cond-mat.mes-hall].

${ }^{32}$ C. Weiss et al., Phys. Rev. Lett. 105, 086103 (2010).

${ }^{33}$ L. Pizzagalli et al., Europhys. Lett. 38, 97 (1997). 